\title{
SOLUTION OF A NON-HOMOGENEOUS LIAPUNOV'S EQUATION
}

\author{
K.N. MURTY AND K.R. PRASAD
}

\section{Introduction}

Boundary value problems play an important role in the theory of differential equations and its applications to physical and technological problems. In finding solutions to two or three or n-point boundary value problems for non-homogeneous problems the construction of Green's matrix is vital.

In this paper we construct the general solution of the non-homogeneous Liapunov system of differential equations by super-imposing the solutions of two non-homogeneous systems. Further, the same principle is applied to find solutions of initial and boundary value problems. In the first case the solution of the problems coincide with the solution we obtained by variation of parameter method [4]. In the case of boundary value problem the method of variation of parameters fails because of the fact that the constant matrix is imbedded by two fundamental matrices.

In section-2, we develop existence and uniqueness criteria for the system of first order matrix differential equation

$$
X^{\prime}(t)=X(t) B(t)+F(t)
$$

where $X(t), B(t)$ and $F(t)$ are square matrices of order $\mathrm{n}$. In section-3, we obtain the Green's matrix for (1.1) satisfying the boundary conditions at three-points of the form

$$
X\left(a_{1}\right) N_{1}+X\left(a_{2}\right) N_{2}+X\left(a_{3}\right) N_{3}=0 .
$$

In this section we also study the properties of Green's matrix. In section-4,we obtain the solution of non-homogeneous Liapunov's differential equation of the form

$$
X^{\prime}(t)=A(t) X(t)+X(t) B(t)+F(t) .
$$

In this section we obtain the solution of problem (1.3) satisfying the solution of the equation

$$
X^{\prime}(t)=A(t) X(t)+F(t)
$$

Received September 30, 1989. 


$$
\text { satisfying } X(a)=\alpha
$$

with the solution of the equation (1.1) satisfying the initial condition matrix (1.5). We also obtain the solution of the boundary value problem (1.3) satisfying the mixed boundary conditions of the form

$$
M_{1} X\left(a_{1}\right)+X\left(a_{1}\right) N_{1}+M_{2} X\left(a_{2}\right)+X\left(a_{2}\right) N_{2}+M_{3} X\left(a_{3}\right)+X\left(a_{3}\right) N_{3}
$$

by superimposing the solution of (1.4) satisfying the boundary conditions

$$
M_{1} X\left(a_{1}\right)+M_{2} X\left(a_{2}\right)+M_{3} X\left(a_{3}\right)=0
$$

with the solution of (1.1) satisfying the boundary conditions (1.2). In section-5, we obtain the adjoint equation for the homogeneous Liapunov's equation.

\section{Existence of solution of the equation (1.1)}

Lemma 2.1. Any solution of the matrix differential equation (1.3) is of the from

$$
X(t)=Y_{1}(t) C_{1}+Y_{1}(t)\left[\int_{a}^{t} Y_{1}^{-1}(s) F(s) d s\right],
$$

where $Y_{1}(t)$ is a fundamental matrix solution of $X^{\prime}(t)=A(t) X(t)$ and $C_{1}$ is a constant square matrix.

Theorem 2.1. Any solution of the equation $X^{\prime}(t)=X(t) B(t)$ is of the form $C_{2}^{*} Y_{2}^{*}(t)$, where $Y_{2}(t)$ is a fundamental matrix solution of $X^{\prime}(t)=B^{*}(t) X(t)$ and $C_{2}$ is a constant square matrix.

Theorem 2.2. A particular solution $\bar{Y}(t)$ of (1.1) is of the form

$$
\bar{Y}(t)=\left[\int_{a}^{t} F(s) Y_{2}^{*-1}(s) d s\right] Y_{2}^{*}(t),
$$

where $Y_{2}(t)$ is a fundamental matrix solution of

$$
X^{\prime}(t)=B^{*}(t) X(t)
$$

Proof. We seek a particular solution of (1.1) in the form $K(t) Y_{2}^{*}(t)$, where $K(t)$ is a square matrix of order $n$. Then it can easily be verified that

$$
K(t)=\int_{a}^{t} F(s) Y_{2}^{*-1}(s) d s
$$

Hence the particular solution of (1.1) is of the form

$$
\bar{Y}(t)=\left[\int_{a}^{t} F(s) Y_{2}^{*-1}(s) d s\right] Y_{2}^{*}(t) .
$$


Theorem 2.3. Any solution of (1.1) is of the form

$$
X(t)=C_{1} Y_{2}^{*}(t)+\bar{Y}(t)
$$

where $\bar{Y}(t)$ is the particular solution of (1.1)

Proof. It can easily be verified that $C_{1} Y_{2}^{*}(t)+\bar{Y}(t)$ is a particular solution of (1.1) for any constant matrix $C_{1}$. Now to prove that every solution is of the form, let $X(t)$ be any solution of (1.1) and $\bar{Y}(t)$ be a particular solution of (1.1). Then it can easily be verifred that $X(t)-\bar{Y}(t)$ is a solution of (2.1). Hence

$$
X(t)-\bar{Y}(t)=C_{1} Y_{2}^{*}(t) \quad \text { or } \quad X(t)=C_{1} Y_{2}^{*}(t)+\bar{Y}(t) .
$$

\section{Three-point boundary value probiem}

In this section we establish existence and uniqueness of solutions to three-point boundary value problem associated with (1.1) satisfying the boundary conditions (1.2) and obtain the properties of Green's matrix.

Definition 3.1. The dimension of the solution space of the boundary value problem is called the index of compatibility of the problem.

Definition 3.2. If $Y_{2}(t)$ is a fundamental matrix solution of the equation $X^{\prime}(t)=$ $B^{*}(t) X(t)$, then the matrix $D_{2}$ defined by

$$
D_{2}=Y_{2}^{*}\left(a_{1}\right) N_{1}+Y_{2}^{*}\left(a_{2}\right) N_{2}+Y_{2}^{*}\left(a_{3}\right) N_{3}
$$

is called the characteristic matrix of the three-point boundary value problem.

Lemma 3.1. Suppose the homogeneous boundary value problem is incompatible. Then there exists a unique solution to the boundary value problem (1.4) satisfying (1.7) and is given by

$$
X(t)=\int_{a_{1}}^{a_{3}} G_{1}(t, s) F(s) d s
$$

where

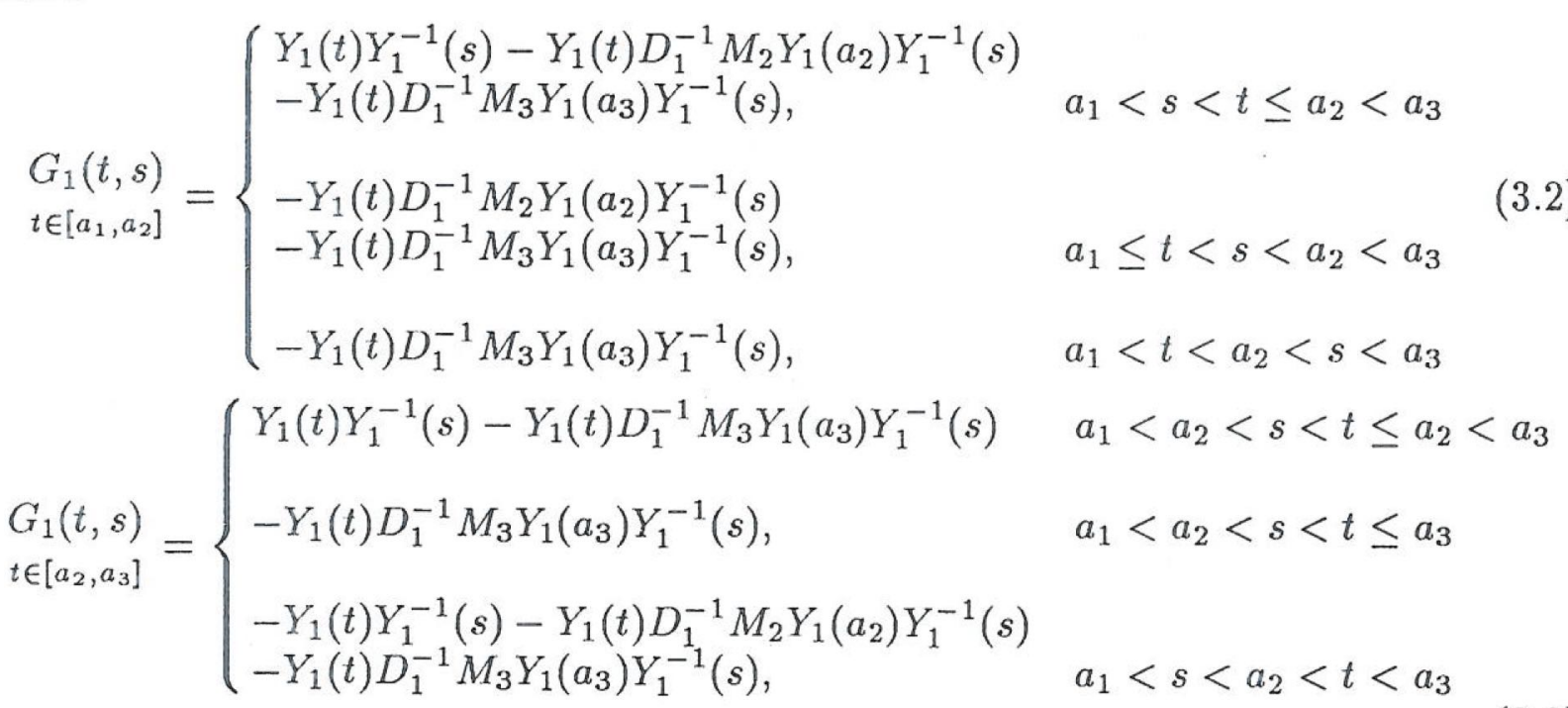


$Y_{1}(t)$ being a fundamental matrix solution of $X^{\prime}(t)=A(t) X(t)$ and $D_{1}$ being the characteristic matrix, and is given by

$$
D_{1}=M_{1} Y_{1}\left(a_{1}\right)+M_{2} Y_{1}\left(a_{2}\right)+M_{3} Y_{1}\left(a_{3}\right)
$$

Theorem 3.1. Suppose the homogeneous boundary value problem is incompatible. There exists a unique solution to the boundary value problem (1.1) satisfying the boundary conditions (1.2) and is given by

$$
X(t)=\int_{a_{1}}^{a_{3}} F(s) G_{2}(t, s) d s
$$

where $G_{2}(t, s)$ is the Green's matrix for the corresponding homogeneous boundary value problem.

Proof. From theorems $2.1,2.2$ and 2.3 any solution of (1.1) is

$$
X(t)=C_{1} Y_{2}^{*}(t)+\left[\int_{a_{1}}^{t} F(s) Y_{2}^{*-1}(s) d s\right] Y_{2}^{*}(t) .
$$

Now to determine the constant matrix $C_{1}$, we substitute the general form of $X(t)$ in the boundary condition matrix (1.2), and get

$$
\begin{aligned}
& C_{1}\left[Y_{2}^{*}\left(a_{1}\right) N_{1}+Y_{2}^{*}\left(a_{2}\right) N_{2}+Y_{2}^{*}\left(a_{3}\right) N_{3}\right]+\left[\int_{a_{1}}^{a_{2}} F(s) Y_{2}^{*-1}(s) d s\right] Y_{2}^{*}\left(a_{2}\right) N_{2} \\
& +\left[\int_{a_{1}}^{a_{3}} F(s) Y_{2}^{*-1}(s) d s\right] Y_{2}^{*}\left(a_{3}\right) N_{3}=0
\end{aligned}
$$

Thus

$$
C_{1}=-\left[\left[\int_{a_{1}}^{a_{2}} F(s) Y_{2}^{*-1}(s) d s\right] Y_{2}^{*}\left(a_{2}\right) N_{2}+\left[\int_{a_{1}}^{a_{3}} F(s) Y_{2}^{*-1}(s) d s\right] Y_{2}^{*}\left(a_{3}\right) N_{3}\right] D_{2}^{-1}
$$

where $D_{2}$ is a characteristic matrix of the boundary value problem. thus

$$
X(t)=\int_{a_{1}}^{a_{3}} F(s) G_{2}(t, s) d s
$$

where

$$
\begin{gathered}
G_{2}(t, s) \\
t \in\left[a_{1}, a_{2}\right]
\end{gathered}= \begin{cases}Y_{2}^{*-1}(s) Y_{2}^{*}(t)-Y_{2}^{*-1}(s) Y_{2}^{*}\left(a_{2}\right) N_{2} D_{2}^{-1} Y_{2}^{*}(t) & \\
-Y_{2}^{*-1}(s) Y_{2}^{*}\left(a_{3}\right) N_{3} D_{2}^{-1} Y_{2}^{*}(t), & a_{1}<s<t \leq a_{2}<a_{3} \\
-Y_{2}^{*-1}(s) Y_{2}^{*}\left(a_{2}\right) N_{2} D_{2}^{-1} Y_{2}^{*}(t) & a_{1} \leq t<s<a_{2}<a_{3} \\
-Y_{2}^{*-1}(s) Y_{2}^{*}\left(a_{3}\right) N_{3} D_{2}^{-1} Y_{2}^{*}(t), & a_{1}<t \leq a_{2}<s<a_{3} \\
-Y_{2}^{*-1}(s) Y_{2}^{*}\left(a_{3}\right) N_{3} D_{2}^{-1} Y_{2}^{*}(t), & \end{cases}
$$




$$
G_{2}(t, s)= \begin{cases}Y_{2}^{*-1}(s) Y_{2}^{*}(t)-Y_{2}^{*-1}(s) Y_{2}^{*}\left(a_{3}\right) N_{3} D_{2}^{-1} Y_{2}^{*}(t), & a_{1}<a_{2}<s<t \leq a_{3} \\ -Y_{2}^{*-1}(s) Y_{2}^{*}\left(a_{3}\right) N_{3} D_{2}^{-1} Y_{2}^{*}(t), & a_{1}<a_{2} \leq t<s \leq a_{3} \\ Y_{2}^{*-1}(s) Y_{2}^{*}(t)-Y_{2}^{*-1}(s) Y_{2}^{*}\left(a_{2}\right) N_{2} D_{2}^{-1} Y_{2}^{*}(t) & \\ -Y_{2}^{*-1}(s) Y_{2}^{*}\left(a_{3}\right) N_{3} D_{2}^{-1}(s) Y_{2}^{*}(t), & a_{1}<s<a_{2}<t<a_{3}\end{cases}
$$

Theorem 3.2. THe Green's matrix has the following properties.

(1) The components of $G_{2}(t, s)$ regarded as a function of $t$ with fixed s, have continuous derivatives every where except at $t=s$. At the point $t=s, G_{2}(t, s)$ has a jump discontinuity of unit magnitude; that is

$$
G_{2}\left(s^{+}, s\right)-G_{2}\left(s^{-}, s\right)=I
$$

(2) $G_{2}$ is a formal solution of the homogeneous boundary value problem (1.1) satisfying (1.2). $G$ fails to be a true solution because of the discontinuity at $t=s$.

(3) $G_{2}(t, s)$ having the properties (1) and (2) is unique.

Proof. The proof (1) is trivial. The representation of $G_{2}(t, s)$ by (3.5) and (3.6) shows that $G_{2}(t, s)$ is a formal solution of (2.1) it fails to be a true solution because of discontinuity at $t=s . G_{2}(t, s)$ satisfies the boundary conditions. For,

$$
\begin{aligned}
& G_{2}\left(a_{1}, s\right) N_{1}+G_{2}\left(a_{2}, s\right) N_{2}+G_{2}\left(a_{3}, s\right) N_{3}, \quad \text { when } s \in\left[a_{1}, a_{2}\right] \\
= & -Y_{2}^{*-1}(s) Y_{2}^{*}\left(a_{2}\right) N_{2} D_{2}^{-1} Y_{2}^{*}\left(a_{1}\right) N_{1}-Y_{2}^{*-1}(s) Y_{2}^{*}\left(a_{3}\right) N_{3} D_{2}^{-1} Y_{2}^{*}\left(a_{1}\right) N_{1} \\
& +Y_{2}^{*-1}(s) Y_{2}^{*}\left(a_{2}\right) N_{2}-Y_{2}^{*-1}(s) Y_{2}^{*}\left(a_{2}\right) N_{2} D_{2}^{-1} Y_{2}^{*}\left(a_{2}\right) N_{2} \\
& \quad-Y_{2}^{*-1}(s) Y_{2}^{*}\left(a_{3}\right) N_{3} D_{2}^{-1} Y_{2}^{*}\left(a_{2}\right) N_{2}+Y_{2}^{*-1}(s) Y_{2}^{*}\left(a_{3}\right) N_{3} \\
& \quad-Y_{2}^{*-1}(s) Y_{2}^{*}\left(a_{2}\right) N_{2} D_{2}^{-1} Y_{2}^{*}\left(a_{3}\right) N_{3}-Y_{2}^{*-1}(s) Y_{2}^{*}\left(a_{3}\right) N_{3} D_{2}^{-1} Y_{2}^{*}\left(a_{3}\right) N_{3} . \\
= & Y_{2}^{*-1}(s)\left[-Y_{2}^{*}\left(a_{2}\right) N_{2} D_{2}^{-1}\left[Y_{2}^{*}\left(a_{1}\right) N_{1}+Y_{2}^{*}\left(a_{2}\right) N_{2}+Y_{2}^{*}\left(a_{3}\right) N_{3}\right]\right. \\
& +Y_{2}^{*}\left(a_{2}\right) N_{2}+Y_{2}^{*}\left(a_{3}\right) N_{3}-Y_{2}^{*}\left(a_{3}\right) N_{3} D_{2}^{-1}\left[Y_{2}^{*}\left(a_{1}\right) N_{1}+Y_{2}^{*}\left(a_{2}\right) N_{2}+Y_{2}^{*}\left(a_{3}\right) N_{3}\right] \\
= & Y_{2}^{*-1}(s)\left[-Y_{2}^{*}\left(a_{2}\right) N_{2}+Y_{2}^{*}\left(a_{2}\right) N_{2}+Y_{2}^{*}\left(a_{3}\right) N_{3}-Y_{2}^{*}\left(a_{3}\right) N_{3}\right] \\
= & 0 .
\end{aligned}
$$

Similarly, we obtain

$$
G_{2}\left(a_{1}, s\right) N_{1}+G_{2}\left(a_{2}, s\right) N_{2}+G_{2}\left(a_{3}, s\right) N_{3}=0
$$

when $s \in\left[a_{2}, a_{3}\right]$.

\section{Nom-homogeneous Liapunov's Equation}

In this section we obtain of the non-homogeneous Liapunov's equation (1.3), satisfying the initial condition (1.5) by superimposing the solutions of initial value problem (1.4) 
satisfying (1.5) with the solution of initial value problem (1.1) satisfying (1.5). Similarly we obtain the solution of (1.3) satisfying the boundary conditions (1.6) by superimposing the solution of boundary value problem (1.4) satisfying (1.7) with the solution of boundary value problem (1.1) satisfying (1.2).

(i) Initail value problem. By lemma 2.1, the solution of (1.4) is

$$
X(t)=Y_{1}(t) C_{1}+Y_{1}(t) \int_{a}^{t} Y_{1}^{-1}(s) F(s) d s .
$$

T'o obtain the constant matrix $C_{1}$, with the initial condition $X(a)=\alpha$, we obtain

$$
X(t)=Y_{1}(t) Y_{1}^{-1}(a) \alpha+Y_{1}(t) \int_{a}^{t} Y_{1}^{-1}(s) F(s) d s .
$$

Similarly, by using theorems $2.1,2.2$ and 2.3 the solution of (1.1) is

$$
X(t)=C_{2} Y_{2}^{*}(t)+\left[\int_{a}^{t} F(s) Y_{2}^{*-1}(s) d s\right] Y_{2}^{*}(t)
$$

To obtain the constant matrix $C_{2}$, with the initial condition $X(a)=\alpha$, we obtain

$$
X(t)=\alpha Y_{2}^{*-1}(a) Y_{2}^{*}(t)+\left[\int_{a}^{t} F(s) Y_{2}^{*-1}(s) d s\right] Y_{2}^{*}(t)
$$

Now by superimposing the two solutions (4.1) and (4.2) we obtain

$$
\begin{aligned}
X(t)= & Y_{1}(t) Y_{1}^{-1}(a) \alpha Y_{2}^{*-1}(a) Y_{2}^{*}(t) \\
& +Y_{1}(t)\left[\int_{a}^{t} Y_{1}^{-1}(s) F(s) Y_{2}^{*-1}(s) d s\right] Y_{2}^{*}(t)
\end{aligned}
$$

which is the solution of the non-homogeneous Liapunov's equation (1.3), satisfying the initial condition (1.5).

(ii) Boundary value problem. By lemma 3.1 the solution of (1.4) satisfying the boundary conditions (1.7) is given by

$$
X(t)=\int_{a_{1}}^{a_{3}} G_{1}(t, s) F(s) d s
$$

where $G_{1}(t, s)$ is given by (3.2) and (3.3). Similarly by theorem 3.1 , the solution of (1.1) satisfying the boundary condition (1.2) is given by

$$
X(t)=\int_{a_{1}}^{a_{3}} F(s) G_{2}(t, s) d s
$$


where $G_{2}(t, s)$ is given by (3.5) and (3.6). Now by superimposing the two solutions (4.3) and (4.4) we obtain

$$
X(t)=\int_{a_{1}}^{a_{3}} G_{1}(t, s) F(s) G_{2}(t, s) d s
$$

where $G_{1}(t, s)$ is given by (3.2) and (3.3) and $G_{2}(t, s)$ is given by (3.5) and (3.6) which may be the solution of the non-homogeneous Liapunov's equation (1.3) satisfying the boundary conditions (1.6).

\section{Adjoint equation for Liapunov's equation}

Consider the homogeneous Liapunov's equation

$$
X^{\prime}=A X+X B
$$

where $X=x_{i j}, A=a_{i j}$ and $B=b_{i j}, i=1,2, \cdots, n ; j=1,2, \cdots, n$. Now the system (5.1) can be written as

$$
\bar{X}^{\prime}=R \bar{X}
$$

where

$$
\bar{X}=\left(\begin{array}{c}
x_{11} \\
x_{12} \\
\vdots \\
x_{1 n} \\
x_{21} \\
x_{22} \\
\vdots \\
x_{2 n} \\
\vdots \\
x_{n 1} \\
x_{n 2} \\
\vdots \\
x_{n n}
\end{array}\right) \text { and } R=\left(\begin{array}{ccccc}
a_{11} I+B^{*} & a_{12} I & a_{13} I & \ldots & a_{1 n} I \\
a_{21} I & a_{22} I+B^{*} & a_{23} I & \cdots & a_{2 n} I \\
\cdots & \cdots & \cdots & \cdots & \cdots \\
\cdots & \cdots & \cdots & \cdots & \cdots \\
\cdots & \cdots & \cdots & \cdots & \cdots \\
a_{n 1} I & a_{n 2} I & a_{n 3} I & \cdots & a_{n n} I+B^{*}
\end{array}\right)
$$

and $\bar{X}$ is, $\left(n^{2} \times 1\right)$ row vector and $R$ is square matrix of order $n^{2}$. Let $L \bar{X}=\bar{X}^{\prime}-R \bar{X}$. It is well known that the adjoint operator of $L$ is $L^{*}$ and is given by

$$
L^{*} Z=\left(-Z^{-1}-R^{*} Z\right)
$$

The adjoint equation for $(5.2)$ is

$$
\bar{Z}^{\prime}=-R^{*} \bar{Z}
$$


where $\bar{Z}=\left(z_{11}, z_{12}, \ldots, z_{1 n}, z_{21}, z_{22}, \ldots, z_{2 n}, \ldots, z_{n 1}, z_{n 2}, \ldots, z_{n n}\right)^{*}$. Thus the system (5.3) can be rewritten as

$$
Z^{\prime}=-A^{*} Z-Z B^{*}
$$

where $Z$ is square matrix of order $n$, and is the adjoint equation for the Liapunov's equation (5.1).

If $A$ and $B$ are skew-symmetric matrices then the system (5.1) is said to be selfadjoint.

\section{References.}

[1] Bellman, R., Introduction to matrix Analysis, Mc-Graw-Hill, New York (1970).

[2] Bellman, R. and Vasudevan, R., "Quasilinearization and matrix Riccati equation," J. Math. Anal. Appl. 64(1978), 106-113.

[3] Cole, R.H. Theory of ordinary differential equations, Appleton-century Crafts, New York (1968).

[4] Murty, K.N., Prasad, B.D.C.N. and Prasad, K.R., "A Note on Matrix Riccati functional differential equation," J. Math. Anal. Appl. 132(1) (1988) 50-56.

Department of Applied Mathematics, Andhra University, Waltair 530003 , India. 